\title{
Proton acceleration and its energy spectra during the coalescence of two cross current loops
}

\author{
J. I. Sakai and K. Shimada \\ Laboratory for Plasma Astrophysics Faculty of Engineering, Toyama University, 3190 Gofuku, Toyama 930-8555, Japan \\ e-mail: sakaijun@eng.toyama-u.ac.jp
}

Received 17 January 2005 / Accepted 7 February 2005

\begin{abstract}
We investigate plasma dynamics during coalescence of two cross current loops by using a resistive three-dimensional MHD simulation code, paying particular attention to finding the most effective electromagnetic fields for producing high-energy protons. Next we investigate the orbit of many protons in the electromagnetic fields obtained from the MHD simulations and find that proton acceleration is more efficient compared with the case of two parallel loops coalescing. It is shown that the maximum proton energy is about $25 \mathrm{MeV}$, which exceeds the energy $(2.223 \mathrm{MeV})$ of the observed prompt nuclear de-excitation lines of gamma-ray and that the proton energy spectrum is a power-law type with an index of about 2-2.3. The simulation results imply that proton-associated gamma-ray sources are located near the footpoints with magnetic north polarity. We found that strong proton acceleration leading to the observed prompt line gamma-ray emissions can be realized only when both poloidal and axial magnetic fields reconnect completely.
\end{abstract}

Key words. Sun: flares - magnetohydrodynamics (MHD) - methods: numerical - acceleration of particles

\section{Introduction}

When energetic protons accelerated during impulsive flares collide with a solar atmosphere, they produce excited nuclei that promptly emit nuclear de-excitation lines, as well as secondary neutrons and positrons, which then results in a delayed 2.223 MeV neutron-capture and $511 \mathrm{KeV}$ positron-annihilation line emission. In most strong flare events the time profile of the prompt gamma-ray line emission is found to be very similar to that of the bremsstrahlung hard X-rays emitted by energetic electrons. This suggests that acceleration and propagation of the flare-accelerated protons and electrons are closely related.

However, the location, size, and geometry of the accelerated proton collision region has remained unknown until now. Recent paper by Hurford et al. (2003) presents the first gamma-ray images of a solar flare taken from the Reuven Ramaty High Energy Solar Spectroscopic Imager (RHESSI) for the X4.8 flare on 23 July 2002. The result shows that the centroid of the $2.223 \mathrm{MeV}$ image was found to be displaced by $20 \pm 6$ arcsec from that of the $0.3-0.5 \mathrm{MeV}$, implying a difference in acceleration and /or propagation between the accelerated electron and proton populations near the Sun. The fact that this proton-associated gamma-ray source does not coincide with the electron-bremsstrahlung sources suggests that the protons would be accelerated in one direction by the DC electric field and could subsequently interact in spatially separated sources.

Sakai \& Shimada (2004) investigated the plasma dynamics during coalescence of two parallel current loops (Tajima et al. 1982; Sakai \& Ohsawa 1987; Sakai \& De Jager 1996;
Aschwanden 2002) by using a resistive three-dimensional MHD simulation code and the orbits of test protons under the most effective electromagnetic fields obtained from the MHD simulations. They found that for the proton acceleration the co-helicity reconnection is more efficient than counterhelicity reconnection. The protons can be accelerated mostly in one direction along the loop axis near where magnetic reconnection occurs. They also showed that the proton energy spectrum is neither a purly power-law type nor purly exponential.

They proposed one possible scenario to explain this recent observation, as follows. The single loop is supposed to be disrupted by a mechanism recently proposed by Sakai et al. (2002), the disrupted part of the loop with its high energy protons, as well as hot thermalized protons, could move up and then interact with other overlying loop. Interaction between the ascending magnetized plasma blobs and the other loop can lead to magnetic reconnection in the interaction region, where the proton could be accelerated more by the inductive electric field associated with magnetic reconnection mostly in one direction along the guiding magnetic field. Saito \& Sakai (2004) investigated the interaction of two current loops with a force-free magnetic field configuration by using $2 \mathrm{D} 3 \mathrm{~V}$, a fully relativistic electromagnetic particle-in-cell code. They found that in a later stage of the coalescence of these two current loops, fast magnetosonic waves are generated as a result of the coalescence rebounding and they develop into shock waves propagating away from the coalesced loops. They also found that near the fast magnetosonic shock front the ions can be promptly accelerated by the surfatron acceleration mechanism. 
Another possible explanation (Emslie et al. 2004) was proposed in terms of stochastic acceleration driven by MHD turbulence cascading, which favors ion acceleration in larger loops and electron acceleration in shorter loops. Therefore it is now important to investigate the proton acceleration processes in details for different types of flares.

In the present paper we first investigate the plasma dynamics during coalescence of two cross current loops (Sect. 2, below) by using a resistive three-dimensional MHD simulation code developed by Sokolov et al. (1999, 2002), paying particular attention to finding the most effective electromagnetic fields for producing high-energy protons (Sect. 3). Next we investigate the orbits of many protons in the electromagnetic fields obtained from the MHD simulations (Sect. 4). In the final section we summarize our results, which found that the proton acceleration is more efficient than the case of two parallel loops coalescencing, as investigated by Sakai \& Shimada (2004). It is shown that the maximum proton energy is about $25 \mathrm{MeV}$, which exceeds the energy $(2.223 \mathrm{MeV})$ of the observed prompt nuclear de-excitation lines of gamma-ray and the proton energy spectrum is a power-law type with an index of about 2-2.3. The simulation results imply that proton-associated gamma-ray sources are located near the footpoints with magnetic north polarity.

\section{Methods of simulations}

In this section we describe two methods of simulation to obtain the energy spectrum of the protons accelerated during coalescence of two cross current loops. Figure 1 shows a schematic picture (lower figure) of two current loops (Loop1 and Loop2) that are crossing with an angle of 90 degrees and the coordinate system (upper figure) used in the simulation.

First we briefly present a model of two cross loops coalescence flare by using a three-dimensional resistive MHD simulation to obtain the electromagnetic fields during the loop coalescence. Next we present the simulation method used to obtain the orbits of many protons under the electromagnetic fields obtained from the MHD simulations.

\subsection{Resistive MHD simulation}

A resistive 3-D MHD code with the recently proposed Artificial Wind (AW) numerical scheme (Sokolov et al. 1999, 2002) splitting over the spatial coordinates is employed. The basis of the AW scheme is that a fundamental physical invariance, Galilean (or more general, Lorentz) invariance, allows the governing equation to be solved in a different steadily moving frame. The principle of the AW scheme is that the frame of reference may be chosen in such a way that the flow under simulation is supersonic there. The problem of upwinding becomes trivial and considerably facilitated versions of discontinuity-capturing schemes may be employed. An extra velocity (Artificial Wind) is added to the velocity of the flow under simulation when the system of coordinates is changed.

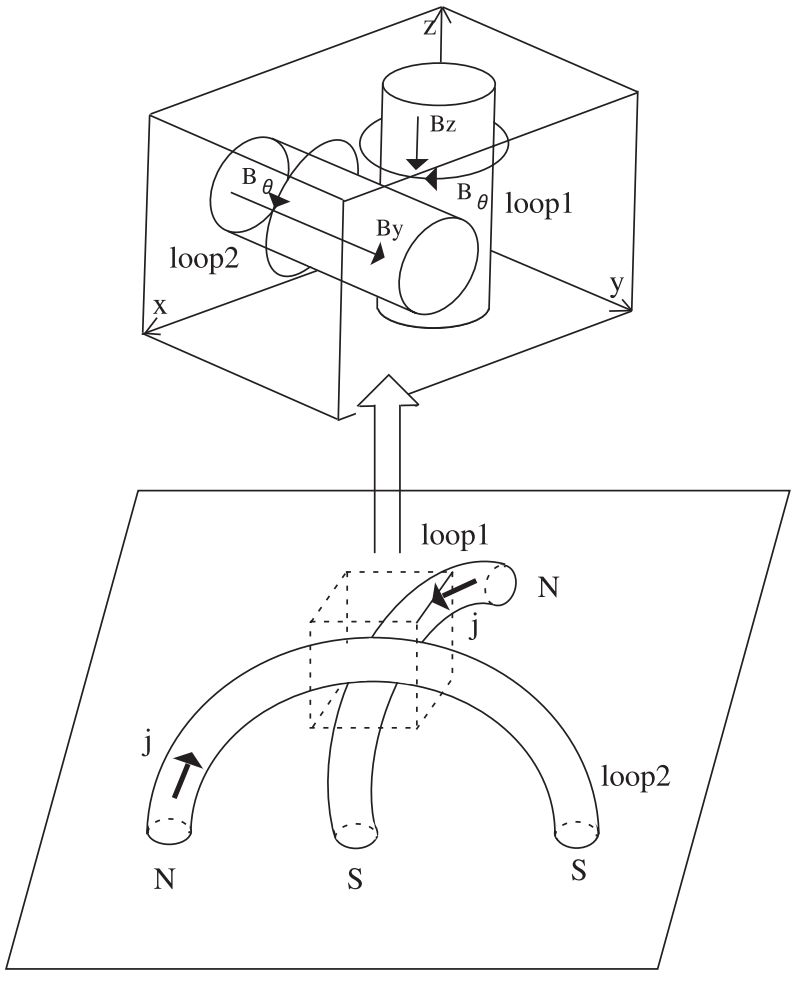

Fig. 1. A schematic picture (lower figure) of two current loops (Loop1 and Loop2) that are crossing with 90 degrees and the coordinate system (upper figure). The system's size is $N_{x}=300, N_{y}=N_{z}=200$.

The MHD equations are numerically solved in a conservative form as follows:

$\frac{\partial \rho}{\partial t}+\frac{\partial}{\partial x_{i}}\left(\rho V_{i}\right)=0$

$\frac{\partial\left(\rho V_{i}\right)}{\partial t}+\frac{\partial}{\partial x_{j}}\left[\rho V_{i} V_{j}+\left(p+B^{2}\right) \delta_{i j}-2 B_{i} B_{j}\right]=0$,

$\frac{\partial B_{i}}{\partial t}+\frac{\partial}{\partial x_{j}}\left(V_{j} B_{i}-V_{i} B_{j}\right)=\frac{1}{R_{m}} \frac{\partial^{2} B_{i}}{\partial x_{j}^{2}}$

$\frac{\partial}{\partial t}\left(\frac{\rho V^{2}}{2}+\frac{p}{\gamma-1}+B^{2}\right)$

$+\frac{\partial}{\partial x_{i}}\left[V_{i}\left(\frac{\rho V^{2}}{2}+\frac{\gamma p}{\gamma-1}+2 B^{2}\right)-2 B_{i} B_{j} V_{j}+Q_{i}\right]=0$

where $\rho, V_{i}, p$, and $B_{i}$ are the density, velocity, pressure, and magnetic field; where $\gamma$ is the adiabatic constant, which is taken to be $\gamma=5 / 3 ; R_{\mathrm{m}}$ the magnetic Reynolds number; $\delta_{i j}$ a unity tensor and $q_{i}$ a dissipative energy flux. The density, pressure, velocity, and magnetic field are normalized by $\rho_{0}, p_{0}, \sqrt{p_{0} / \rho_{0}}$, and $B_{0}=\sqrt{8 \pi p_{0}}$, respectively.

For resistive MHD with a large but finite value of $R_{\mathrm{m}}$, the energy equation Eq. (4) should be obtained as a sum of the equation for plasma energy, in which the Joule heating term is 
present as follows: $\frac{(c \nabla \times \boldsymbol{B})^{2}}{(4 \pi)^{2} \sigma}$ and the equation for magnetic energy which is given by the Eq. (4) multiplied by the magnetic field $B_{i} /(4 \pi)$. All the variables are not normalized here, and $\sigma$ is a conductivity. The dissipation of the magnetic field energy is given by the term as follows: $\frac{c^{2}}{(4 \pi)^{2} \sigma} B_{i} \Delta B_{i}$. So in the Eq. (4) for the total energy, the Joule heating is compensated for by magnetic energy dissipation in the following way:

$\frac{c^{2}}{(4 \pi)^{2} \sigma}\left[B_{i} \Delta B_{i}+(\nabla \times \boldsymbol{B})^{2}\right]=\frac{c^{2}}{(4 \pi)^{2} \sigma} \nabla \cdot[\boldsymbol{B} \times \nabla \times \boldsymbol{B}]$.

As a result, the resistive dissipation in the energy equation is present only in the form of the additional dissipative energy flux, which in normalized variables, may be written as follows: $Q_{i}^{(m)}=R_{\mathrm{m}}^{-1} \frac{\partial}{\partial x_{j}}\left(2 B_{i} B_{j}-B^{2} \delta_{i j}\right)$. The dissipative energy flux due to heat transfer may also be taken into account in its usual form: $Q_{i}^{(h)}=\lambda_{i j} \frac{\partial}{\partial x_{j}} \frac{p}{\rho}$. For the sake of simplicity here we fully neglect the non-diagonal part of dissipative energy transfer tensor and substitute all the tensors in $q_{i}$ for unity tensors: $B_{i} B_{j}=B^{2} \delta_{i j} / 3$, $\lambda_{i j}=\lambda_{l l} \delta_{i j} / 3$. This numerical simulation shows that the influence of the dissipative energy flux is insignificant, so we do not try to take it more carefully into account. Finally we admit the dissipative hydrodynamic flux to be as follows:

$Q_{i}=\frac{\partial}{\partial x_{i}}\left(-\frac{B^{2}}{3 R_{\mathrm{m}}}-\lambda \frac{p}{\rho}\right)$

The magnetic Reynolds number $R_{\mathrm{m}}=1.3 \times 10^{3}$ and the heat transfer constant $\lambda=2.5 \times 10^{-4}$ are used in simulation. Magnetic reconnection takes place through finite conductivity, while conductivity due to microturbulence (Vasyliunas 1975) may be written by the expression

$\sigma=\omega_{\mathrm{pe}}^{2} / 4 \pi v_{\mathrm{eff}}$,

where $v_{\mathrm{eff}}$ is the effective collision frequency between some high-frequency turbulent fields and the electrons. For strong turbulence driven in the current sheet due to electric currents like Buneman-type instability the effective collision frequency is of the order of the electron plasma frequency, namely $v_{\mathrm{eff}} \approx$ $\omega_{\text {pe }}$. Therefore, if we take the typical density in the corona as $10^{8}, c_{\mathrm{s}} \approx 10^{7} \mathrm{~cm} \mathrm{~s}^{-1}$, and $L \approx 10^{7} \mathrm{~cm}$, we obtain $R_{\mathrm{m}} \approx 10^{3-4}$. Radiating boundary conditions where all physical quantities are linearly extrapolated at the boundary were used for all directions.

Here we take a system size of $N_{x}=300$ and $N_{y}=N_{z}=200$, as shown in Fig. 1. The two current loops, where Loop1 is located parallel along the $z$-axis, while Loop2 is located parallel along the $y$-axis. Both loops are assumed to be in an equilibrium state, in which the magnetic field and pressure for each loop are given as

$$
\begin{aligned}
& B_{x}=q_{2} B_{02} \frac{\left(z-z_{c 2}\right)}{a} \mathrm{e}^{-\left(\frac{r_{2}}{a}\right)^{2}}+q_{1} B_{01} \frac{\left(y-y_{c 1}\right)}{a} \mathrm{e}^{-\left(\frac{r_{1}}{a}\right)^{2},} \\
& B_{y}=B_{02} \mathrm{e}^{-\left(\frac{r_{2}}{a}\right)^{2}}-q_{1} B_{01} \frac{\left(x-x_{c 1}\right)}{a} \mathrm{e}^{-\left(\frac{r_{1}}{a}\right)^{2},} \\
& B_{z}=B_{01} \mathrm{e}^{-\left(\frac{r_{1}}{a}\right)^{2}}-q_{2} B_{02} \frac{\left(x-x_{c 2}\right)}{a} \mathrm{e}^{-\left(\frac{r_{2}}{a}\right)^{2}},
\end{aligned}
$$

$$
p_{i}=\left(\frac{q_{i}^{2}}{2}-\frac{q_{i}^{2} r_{i}^{2}}{a^{2}}-1\right) \mathrm{e}^{-2\left(\frac{r_{i}}{a}\right)^{2}}+0.55,
$$

where $r_{1}=\sqrt{\left(x-x_{c 1}\right)^{2}+\left(y-y_{c 1}\right)^{2}}, r_{2}=\left(\left(x-x_{c 2}\right)^{2}+\right.$ $\left.\left(z-z_{c 2}\right)^{2}\right)^{1 / 2}$, and the centers of the two flux tubes with radius $a=30$ are $\left(x_{c 1}, y_{c 1}\right)=(105,100)$ and $\left(x_{c 2}, z_{c 2}\right)=(195,100)$. The density profile is the same as the pressure. Twist parameter $q_{i}$ is $q_{1}=q_{2}=1$ and $B_{01}=-1$ and $B_{02}=1.0$. The plasma beta $\beta$ in the center of the two loops is 0.06 .

\subsection{Simulation of proton dynamics}

By using the electromagnetic fields obtained from the MHD equations above during coalescence of two parallel current loops, we investigate the protons dynamics to obtain their energy spectrum. The normalized relativistic equations of the motion of a proton is given by

$$
\begin{aligned}
& \frac{\mathrm{d} \boldsymbol{u}}{\mathrm{d} t}=\boldsymbol{E}+\frac{\boldsymbol{u} \times \boldsymbol{B}}{\gamma}, \\
& \frac{\mathrm{d} \boldsymbol{x}}{\mathrm{d} t}=\frac{R \boldsymbol{v}}{\gamma},
\end{aligned}
$$

where proton velocity $\boldsymbol{v}=\gamma^{-1} \boldsymbol{u}$, electric field $\boldsymbol{E}$, and magnetic field $\boldsymbol{B}$ are normalized by Alfvén velocity $V_{\mathrm{A}}, E_{0}=V_{\mathrm{A}} B_{0} / c$, and $B_{0}$, respectively. The time is normalized by proton cyclotron frequency $\omega_{c i}$, and the length is normalized by the distance over which the fields are changed across the loop radius $(a)$. The Lorentz factor $\gamma$ is given by $\gamma=\left(1+A^{2} u^{2}\right)^{1 / 2}$, where $A=V_{\mathrm{A}} / c$. Parameter $R$ in Eq. (3) is defined by $R=$ $V_{\mathrm{A}} /\left(\omega_{c i} a\right)$. In our simulation we take $A=1 / 300$ and $R=10^{-8}$.

To advance particles under the guidance of electric and magnetic fields through solving the motion equation numerically, we used a centered-difference form of the NewtonLorentz equations of motions (Buneman 1993; Birdsall \& Langdon 1991), namely

$\boldsymbol{u}^{\text {new }}-\boldsymbol{u}^{\text {old }}=\frac{q \delta t}{m}\left[\boldsymbol{E}+\frac{1}{2 \Gamma}\left(\boldsymbol{u}^{\text {new }}+\boldsymbol{u}^{\text {old }}\right) \times \boldsymbol{B}\right]$,

where $q$ is the charge of a particle, $m$ the mass of a particle, and $\delta t$ the calculate time step. As it stands, the equation for $\boldsymbol{u}^{\text {new }}$ is implicit. We choose the method proposed by Boris (1970) to obtain a simpler explicit solution in several steps.

The protons are randomly distributed with one proton per cell. The initial velocity distribution function for protons is Maxwellian with the thermal velocity $v_{\text {th }}=0.4 V_{\mathrm{A}}$. The simulation time step is $\omega_{c i} \delta t=0.05$. The boundary conditions for protons are open in the $x$-, $y$-, and $z$-directions.

\section{Simulation results of a two cross loop coalescence}

The basic physical evolution of reconnection between two cross loops was investigated by several authors (see Sakai 1990b; Sakai \& de Jager 1997; Linton et al. 2001). Each of the two cross current loops is in a state of equilibrium, as long as the distance between them is much greater than loop radius $a$. 



Fig. 2. Time evolution of the isosurface of total magnetic field intensity with $|\boldsymbol{B}|=0.26$ during the collision of two current loops: a) $t=0$; b) $t=35 \tau_{\mathrm{A}}$; c) $t=59 \tau_{\mathrm{A}}$; and d) $t=82 \tau_{\mathrm{A}}$.

While the loops approach each other, they are no longer in equilibrium and the whole non-equilibrium system tends to a new equilibrium state. Due to attraction, the two initially static current loops begin to move and approach each other. Then they meet and merge with sling-shot magnetic reconnection. We mention here that only when complete magnetic reconnection occurs where both poloidal and axial magnetic fields reconnect, high energy protons are generated.

Here we present the simulation results from the dynamics of two cross current loops, especially paying attention to where and at which stage the strongest electric fields are formed to accelerate protons. Figure 2 shows the time evolution of the isosurface of total magnetic field intensity with $|\boldsymbol{B}|=0.26$ during the collision of two cross current loops: (a) $t=0$; (b) $t=35 \tau_{\mathrm{A}}$; (c) $t=59 \tau_{\mathrm{A}}$; and (d) $t=82 \tau_{\mathrm{A}}$. As shown later, the strongest electric field appears at $t=59 \tau_{\mathrm{A}}$ as shown in Fig. $2 \mathrm{c}$ where magnetic reconnection occurs. Therefore we investigate the spatial structures of the electric and magnetic fields at $t=59 \tau_{\mathrm{A}}$ shown in Fig. 2c.

Figure 3 shows the spatial structures of the electromagnetic fields at $t=59 \tau_{\mathrm{A}}$ in Fig. $2 \mathrm{c}$, which are used in the calculation of test proton orbits. Figure 3a shows $B_{x}$ (gray scale) and vector plot of $B_{y}-B_{z}$ on $x=150$. Figure $3 \mathrm{~b}$ shows $B_{y}$ (gray scale) and vector plot of $B_{x}-B_{z}$ on $y=100$. Figure $3 \mathrm{c}$ shows $E_{x}$ (gray scale) and vector plot of $E_{y}-E_{z}$ on $x=150$. Figure $3 \mathrm{~d}$ shows $E_{y}$ (gray scale) and vector plot of $E_{x}-E_{z}$ on $y=100$.

The most important electric field leading to proton acceleration is the parallel component to the local magnetic field that is generated near the region where magnetic reconnection occurs due to finite resistivity. To find such a region, we calculate the spatial value of the scalar product $\boldsymbol{E} \cdot \boldsymbol{B}$. Figure 4 a shows the spatial value of the scalar product $\boldsymbol{E} \cdot \boldsymbol{B}$ in the $x-z$ plane on $y=100$ at $t=59 \tau_{\mathrm{A}}$. Figure $4 \mathrm{~b}$ shows the spatial value of the scalar product $\boldsymbol{E} \cdot \boldsymbol{B}$ in the $y-z$ plane on $x=150$ at $t=59 \tau_{\mathrm{A}}$.
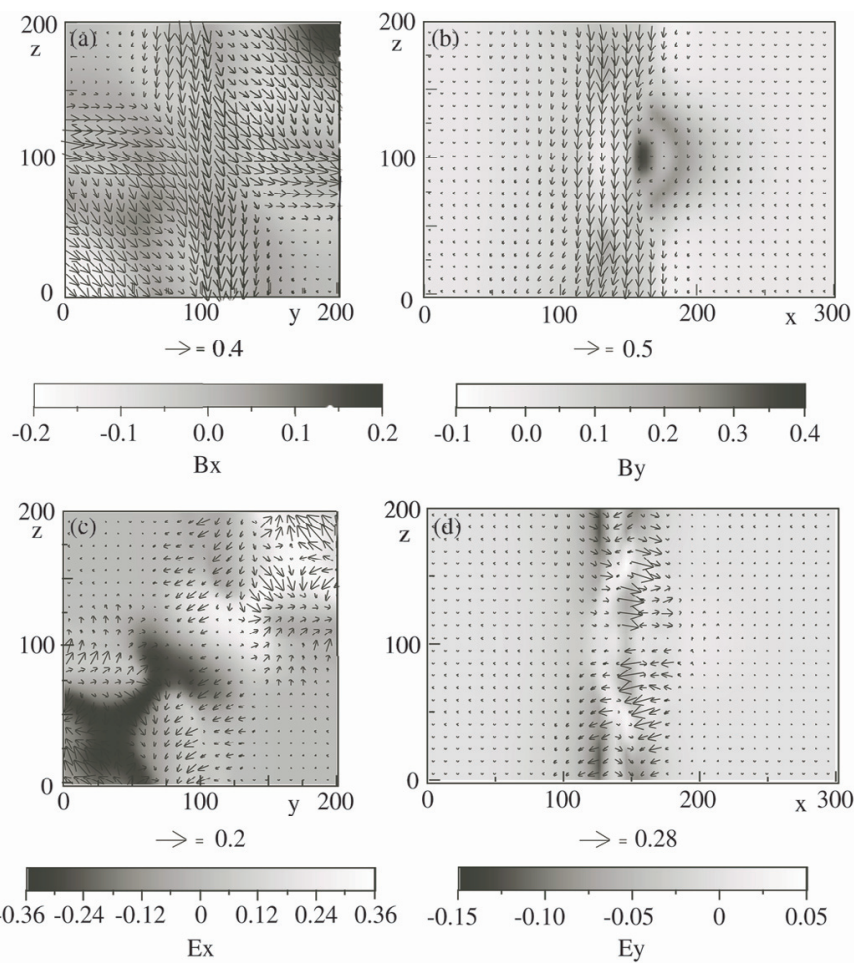

Fig. 3. Spatial structures of the electromagnetic fields at $t=59 \tau_{\mathrm{A}}$ in Fig. $2 \mathrm{c}$ which are used in calculation of test proton orbits. a) $B_{x}$ (gray scale) and vector plot of $B_{y}-B_{z}$ on $x=150$. b) $B_{y}$ (gray scale) and vector plot of $B_{x}-B_{z}$ on $y=100$. c) $E_{x}$ (gray scale) and vector plot of $E_{y}-E_{z}$ on $x=150$. d) $E_{y}$ (gray scale) and vector plot of $E_{x}-E_{z}$ on $y=100$.

The black region shows that the induced electric field is almost parallel to the local magnetic field, while the white region shows the region where the electric field is perpendicular to the local magnetic field.

Therefore we conclude from the MHD simulation that near the magnetic reconnection region strong longitudinal electric field appears along the local magnetic field that may play an important role in the proton acceleration.

\section{Proton acceleration and its energy spectrum}

In this section we present simulation results of the dynamics of many test protons under the MHD electromagnetic fields at $t=59 \tau_{\mathrm{A}}$ discussed in the previous section. First of all we present the proton energy spectra in Fig. 5, where energy $E$ is normalized as $E=\left(V_{x}^{2}+V_{y}^{2}+V_{z}^{2}\right) / V_{\mathrm{A}}^{2}$.

Figure 5a shows the proton energy spectra at $\omega_{c i} t=500$ where the power law index is about 3.6 in the high energy region. Figure $5 \mathrm{~b}$ shows the proton energy spectra at $\omega_{c i} t=1500$, where the power law index is about 2.3 in the high energy region. Figure 5c shows the energy spectra at $\omega_{c i} t=2500$, where the power law index is about 2.0 in the high energy region. If we take the Alfvén velocity as about $1000 \mathrm{~km}$, then the energy $E=10^{2}, 10^{3}$ correspond to $1 \mathrm{MeV}$ and $10 \mathrm{MeV}$, respectively. The maximum proton energy reaches about $25 \mathrm{MeV}$ at $\omega_{c i} t=2500$. 

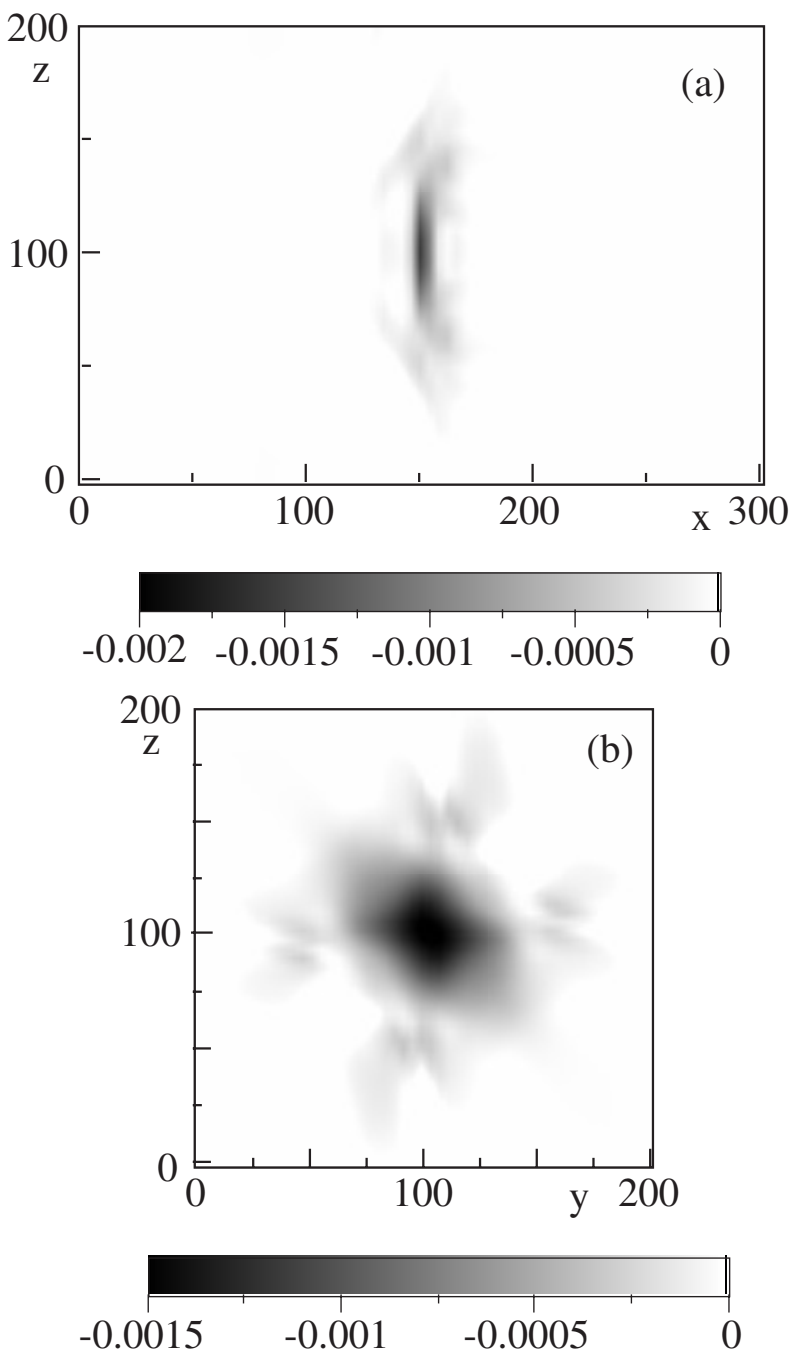

Fig. 4. a) The spatial value of the scalar product $\boldsymbol{E} \cdot \boldsymbol{B}$ in the $x-z$ plane on $y=100$ at $t=59 \tau_{\mathrm{A}}$. b) The spatial value of the scalar product $\boldsymbol{E} \cdot \boldsymbol{B}$ in the $y-z$ plane on $x=150$ at $t=59 \tau_{\mathrm{A}}$. The black region shows that the induced electric field is almost parallel to the local magnetic field, while the white region shows the region where the electric field is perpendicular to the local magnetic field.

To see the direction of proton acceleration, we show the proton velocity distribution functions at $\omega_{c i} t=2500$ in Fig. 6, where Figs. 6a-c show the $x$-direction, $y$-direction, and $z$-direction, respectively. As seen in Fig. 6b, protons in Loop 2 can be accelerated in the negative $y$-direction, while protons in Loop 1 can be accelerated in the positive $z$-direction, as in Fig. 6c.

The reason for protons in the loops being accelerated in one direction is investigated as follows. In Fig. 7 we show the phase space plots of protons at $\omega_{c i} t=2500$, where Fig. 7a shows the plot of $x-V_{y}$ and Fig. 7b shows the plot of $z-V_{y}$. Figure $7 \mathrm{c}$ then shows the spatial structure of electric field $E_{y}$ (gray scale) and the vector plot of $E_{x}-E_{z}$ on $y=100$, where the elliptic region corresponding to Fig. 4 a shows the region of Loop 2 where the proton acceleration is dominant in the negative $y$-direction. In Fig. 8 we show the phase space plots of protons at $\omega_{c i} t=2500$, where Fig. 8a shows the plot of $y-V_{y}$, Fig. $8 \mathrm{~b}$ the plot of $z-V_{y}$, and Fig. $8 \mathrm{c}$ shows the spatial structure

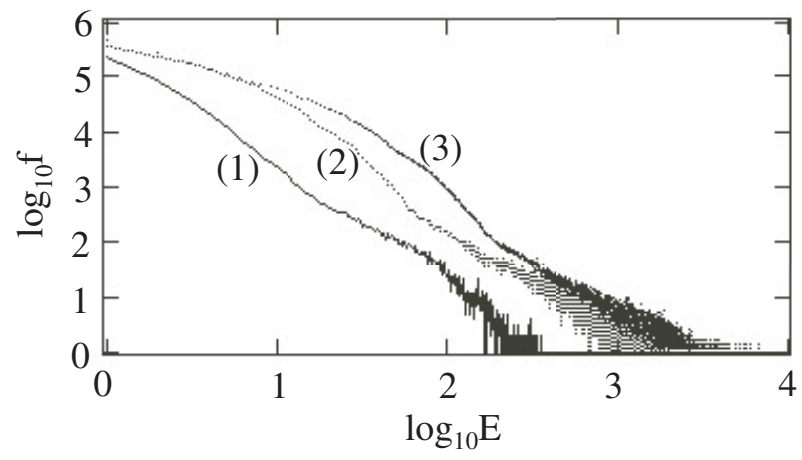

Fig. 5. Proton energy spectra: (1) at $\omega_{c i} t=500$ where the power law index is about 3.6 in the high energy region; (2) at $\omega_{c i} t=1500$, where the power law index is about 2.3 in the high energy region; (3) at $\omega_{c i} t=2500$, where the power law index is about 2.0 in the high energy region.

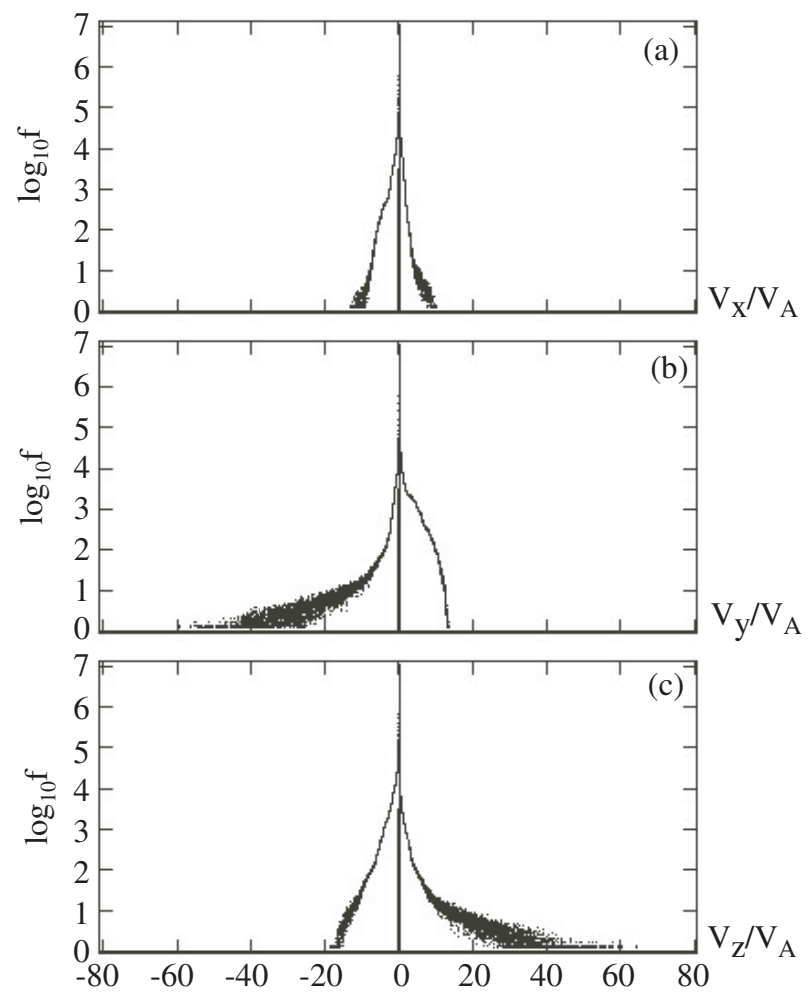

Fig. 6. Proton velocity distribution functions of a) the $x$-direction; b) $y$-direction; and c) $z$-direction at $\omega_{c i} t=2500$. The protons in Loop 2 can be accelerated to the negative $y$-direction, while the protons in Loop 1 can be accelerated to the positive $z$-direction.

of electric field $E_{x}$ (gray scale) and the vector plot of $E_{y}-E_{z}$ on $x=150$, where the elliptic region corresponding to Fig. 4b shows the region where proton acceleration is dominant in the negative $y$-direction. As seen in the elliptic region, the electric field vectors dominante in the negative $y$-direction. From the above analysis we conclude that protons in Loop 2 are accelerated mostly in the negative $y$-direction.

Next we examine the protons in Loop 1. Figure 9 shows the phase space plots of protons at $\omega_{c i} t=2500$, where Fig. 9a shows the plot of $x-V_{z}$ and Fig. $9 \mathrm{~b}$ shows the plot of $y-V_{y}$. Figure 9c shows the spatial structure of electric field, $E_{z}$ (gray 

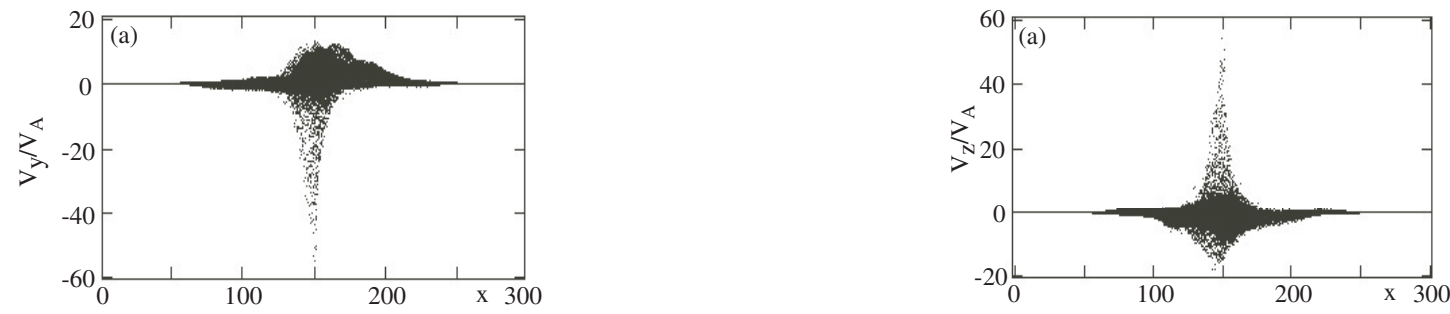
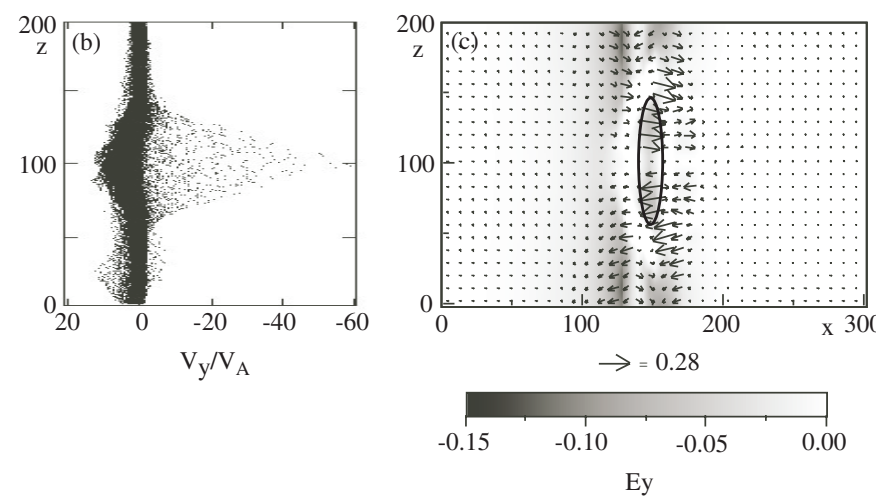

Fig. 7. Phase space plots of protons at $\omega_{c i} t=2500$ : a) $x-V_{y}$; and b) $z-V_{y}$. c) The spatial structure of the electric field, $E_{y}$ (gray scale) and the vector plot of $E_{x}-E_{z}$ on $y=100$, where the elliptic region corresponding to Fig. 4a shows that proton acceleration is dominant.
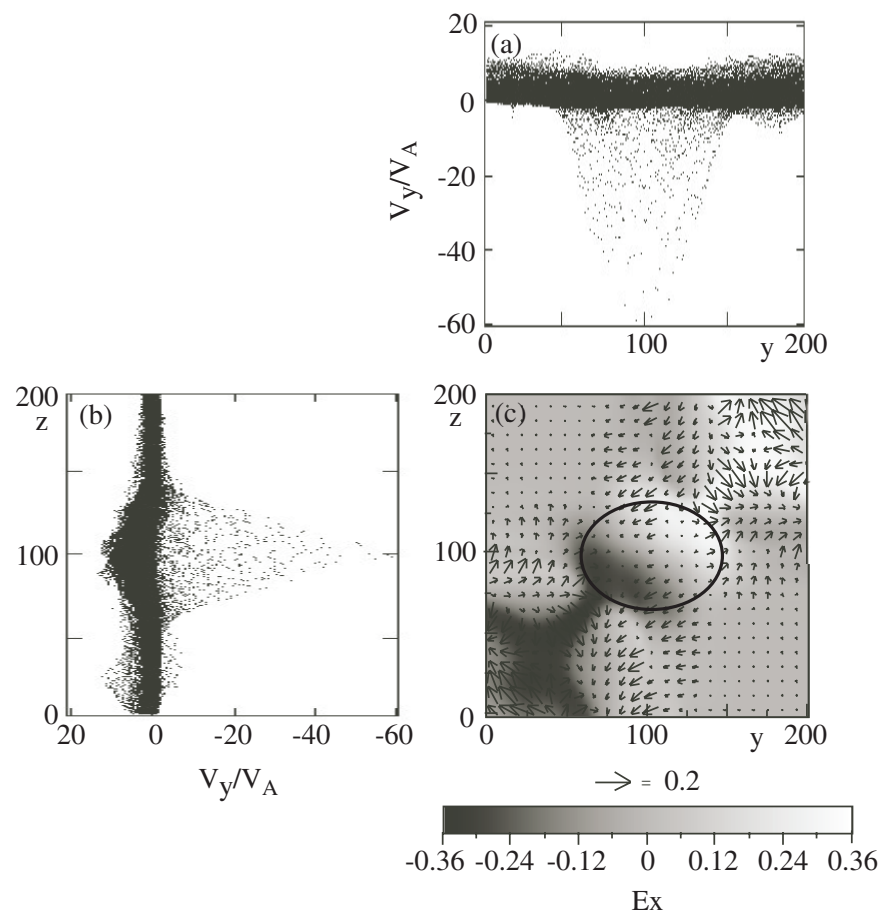

Fig. 8. Phase space plots of protons at $\omega_{c i} t=2500$ : a) $y-V_{y}$; and b) $z-V_{y}$. c) The spatial structure of the electric field, $E_{x}$ (gray scale) and the vector plot of $E_{y}-E_{z}$ on $x=150$, where the elliptic region corresponding to Fig. $4 \mathrm{~b}$ shows that proton acceleration is dominant.

scale) and the vector plot of $E_{x}-E_{y}$ on $z=100$, where the elliptic region corresponding to Fig. 4 a shows the region of Loop 1 where the proton acceleration is dominant in the positive $z$-direction. Figure 10 shows the phase space plots of protons at $\omega_{c i} t=2500$, where Fig. 10a shows the plot of $y-V_{z}$ and


Fig. 9. Phase space plots of protons at $\omega_{c i} t=2500$ : a) $x-V_{z}$; and b) $y-V_{y}$. c) The spatial structure of the electric field, $E_{z}$ (gray scale) and the vector plot of $E_{x}-E_{y}$ on $z=100$, where the elliptic region corresponding to Fig. 4a shows that proton acceleration is dominant.
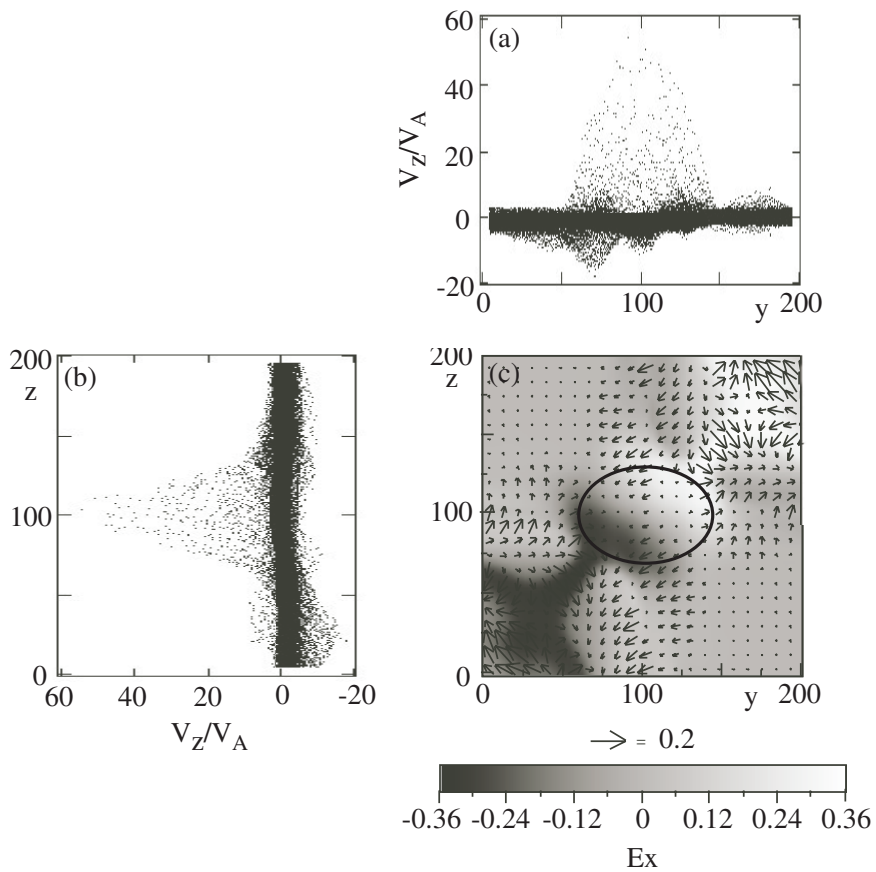

Fig. 10. Phase space plots of protons at $\omega_{c i} t=2500$ : a) $y-V_{z}$; and b) $z-V_{z}$. c) The spatial structure of the electric field, $E_{x}$ (gray scale) and the vector plot of $E_{y}-E_{z}$ on $x=150$, where the elliptic region corresponding to Fig. $4 \mathrm{~b}$ shows that proton acceleration is dominant.

Fig. $10 \mathrm{~b}$ the plot of $z-V_{z}$. Figure 10c shows the spatial structure of electric field $E_{x}$ (gray scale) and the vector plot of $E_{y}-E_{z}$ on $x=150$, where the elliptic region corresponding to Fig. $4 \mathrm{~b}$ shows the region where proton acceleration is dominant in the positive $z$-direction along Loop 1. From the above analysis we 
conclude that the protons in Loop 1 are accelerated mostly in the positive $z$-direction.

We hereby obtain an important result about the direction of proton acceleration during the coalescence of two cross loops, as shown in Fig. 1. The proton-associated gamma-ray sources are located near two footpoints with magnetic north polarity. If one of the axial currents along the loops flows in the opposite direction, then the magnetic reconnection becomes very weak and results in weak proton acceleration. If Loop 1 has a different magnetic polarity from the case shown in Fig. 1, i.e. if the axial magnetic field $B_{z}$ in Loop 1 takes the opposite direction, then magnetic reconnection occurs only for the poloidal components of both current loops. Therefore the electromagnetic fields also becomes weak during coalescence, resulting in the lack of strong proton acceleration generating the observed line gamma-ray emissions. In summary strong proton acceleration leading to the observed prompt line gamma-ray emissions can be realized only when complete magnetic reconnection occurs when both poloidal and axial magnetic fields reconnect.

\section{Conclusions}

We have investigated the behavior of protons near the magnetic reconnection region during coalescence of two cross loops. The electromagnetic fields during the coalescence process were calculated from a three-dimensional resistive MHD simulation, in order to find the most effective electric fields for proton acceleration. According to the result by Mori et al. (1998), who showed that the energy spectrum of accelerated protons near X-type magnetic reconnection regions is universal with power-law spectrum $E^{-\gamma}$ where the power-law index $\gamma$ is about 2.0-2.2, the proton energy spectrum is power-law type with index of about 2-2.3. We found that proton acceleration is more efficient compared to the case of two parallel loops coalescing (Sakai \& Shimada 2004). It was shown that the maximum proton energy reaches about $25 \mathrm{MeV}$ and exceeds the energy
$(2.223 \mathrm{MeV})$ of the observed prompt nuclear de-excitation lines of gamma-ray. The most striking result is that protonassociated gamma-ray sources are located near the footpoints with magnetic north polarity. We have found that strong proton acceleration leading to the observed prompt line gamma-ray emissions can be realized only when both poloidal and axial magnetic fields completely reconnect.

Acknowledgements. We thank the anonymous referee for constructive comments that improved our original paper.

\section{References}

Aschwanden, M. J. 2002, Space Sci. Rev., 101 (1-2), 1

Birdsall, C. K., \& Langdon, A. B. 1991, Plasma Physics via Computer Simulation (Adam Hilger), 58

Boris, J. P. 1970, Proc. Fourth Conf. Num. Sim. Plasmas, Naval Res. Lab., Wash. DC, 3, 2-3 November

Buneman, O. 1993, in Computer Space Plasma Physics, Simulation Techniques and Software, ed. H. Matsumoto, \& Y. Omura (Tokyo: Terra Scientific), 67

Emslie, A. G., Miller, J. A., \& Brown, J. C. 2004, ApJ, 602, L69

Hurford, G. J., Schwartz, R. A., Krucker, S., et al. 2003, ApJ, 595, L77

Linton, M. G., Dahlburg, R. B., \& Antiochos, S. K. 2001, ApJ, 553, 905

Mori, K., Sakai, J. I., \& Zhao, J. 1998, ApJ, 494, 430

Saito, S., \& Sakai, J. I. 2004, ApJ, 604, L133

Sakai, J. I., \& de Jager, C. 1996, Space Sci. Rev., 77, 1

Sakai, J. I., Nishi, K., \& Sokolov, I. V. 2002, ApJ, 576, 519

Sakai, J. I., \& Ohsawa, Y. 1987, Space Sci. Rev., 46, 113

Sakai, J. I., \& Shimada, K. 2004, A\&A, 426, 333

Sokolov, I. V., Timofeev, E. V., Sakai, J. I., \& Takayama, K. 1999, Shock Waves, 9, 423

Sokolov, I. V., Timofeev, E. V., Sakai, J. I., \& Takayama, K. 2002, J. Comp. Phys., 181, 354

Tajima, T., Brunel, F., \& Sakai, J. I. 1982, ApJ, 245, L45

Vasylinuas, V. M. 1975, Geophys. Space Phys., 13, 303 